\title{
Benefits of anti-inflammatory therapy in the treatment of ischemia/reperfusion injury in the renal microvascular endothelium of rats with return of spontaneous circulation
}

\author{
WEI WEI ${ }^{*}$, YONG XIE* ${ }^{*}$, SHI-CHAO LAI, BO-FU LIU, YA-RONG HE, HAI HU and YU CAO \\ Department of Emergency Medicine, West China Hospital of Sichuan University, Chengdu, Sichuan 610041, P.R. China
}

Received February 15, 2016; Accepted February 14, 2017

DOI: $10.3892 / \mathrm{mmr} .2017 .6548$

\begin{abstract}
The aim of the present study was to investigate whether prostaglandin E1 (PGE1), target temperature management (TTM) and a combined intervention involving the two would be beneficial as anti-inflammatory therapies for ischemia/reperfusion (I/R) injury to the renal microvascular endothelium of rats with return of spontaneous circulation (ROSC). In each group of rats with different interventions, following successful cardiopulmonary resuscitation, the levels of thrombomodulin (TM), interleukin-6 (IL-6) and tumor necrosis factor- $\alpha$ (TNF- $\alpha)$ in the plasma were evaluated. The expression of vascular endothelial (VE)-cadherin and vascular cell adhesion molecule-1 (VCAM-1) mRNA was analyzed in the kidney. Hematoxylin and eosin staining and VE-cadherin/vascular endothelial growth factor receptor double fluorescent immunohistochemistry staining were also performed. PGE1 improved tubular cell swelling and inflammatory cell infiltration. PGE1 also alleviated VE-cadherin protein loss in renal microvascular endothelium cells (RMECs), lowered the tubular injury score, decreased VE-cadherin and VCAM-1 mRNA expression, and markedly inhibited the release of TM (at 3 time points) and TNF- $\alpha$ (4 and $8 \mathrm{~h} ; \mathrm{P}<0.05)$. In addition to improving the renal tubular injury score and altering the concentration of TNF- $\alpha$ at $8 \mathrm{~h}$, the effect of TTM was the same as PGE1 for the other indicators $(\mathrm{P}>0.05)$. The PGE1/TTM combined intervention significantly reduced IL-6 concentration at $8 \mathrm{~h}(\mathrm{P}<0.05)$. The correlation analysis demonstrated that the peak TM and TNF- $\alpha$ levels $(\mathrm{P}<0.001, \mathrm{r}=0.809)$, and IL-6 levels $(\mathrm{P}<0.001$, $\mathrm{r}=0.792)$ were positively associated. PGE1 and TTM had a
\end{abstract}

Correspondenceto:DrYuCao,DepartmentofEmergency Medicine, West China Hospital of Sichuan University, 37 Guo Xue Xiang, Chengdu, Sichuan 610041, P.R. China

E-mail:hx112ww@163.com

*Contributed equally

Key words: return of spontaneous circulation, renal microvascular endothelium cell, ischemic/reperfusion injury, inflammation, prostaglandin E1, temperature management protective effect against I/R injury to the RMEC, while the PGE1/TTM combined intervention exhibited an increased synergistic effect as an anti-inflammatory treatment when compared with either of the single interventions.

\section{Introduction}

The mortality rate for out-of-hospital cardiac arrest (OHCA) is as high as $89 \%$. Even if return of spontaneous circulation (ROSC) is successfully achieved, $50 \%$ of patients succumb to the disease prior to hospital discharge (1). Ischemia/reperfusion (I/R) injury is a major concern in patients with cardiac arrest (CA). Endothelial cells are widely distributed and actively secrete multiple factors, including nitric oxide, endothelin 1 and prostacyclin, which are associated with vascular tension, blood coagulation and inflammation. Therefore, the endothelium has been recognized as an excellent target within the signal transduction mechanism of a number of diseases, and subsequently as a key therapeutic target for I/R injury (2). During the reperfusion phase, the release of oxygen free radicals, cytokines, coagulants and complement-activation products leads to marked activation of the inflammatory response, with neutrophil adhesion to the endothelium and the induction of whole body I/R injury (3).

A novel approach for post-CA therapies may improveclinical outcomes and so it has become one of the most important areas of focus in resuscitation science. The post-resuscitation abnormalities following CA observed are similar to the immunological and coagulation disorders exhibited in sepsis. Further investigations into anti-inflammation therapeutic approaches are required for patients following successful resuscitation. Previous studies have suggested that prostaglandin E1 (PGE1) may have anti-inflammatory roles due to weakening of leukocyte adhesion to the microvascular endothelium and lower tissue expression of some inflammatory markers involved in the I/R injury process (4-8). Currently, TTM is recommended as one of the therapeutic strategies for patients with ROSC following CA by the American Heart Association (9). However, it is unclear whether anti-inflammatory effects comprise one of the main protective mechanisms of TTM for PCAS (10-12).

The aim of the present study was to investigate the potential benefits of PGE1 and TTM, with respect to anti-inflammatory effects, for I/R injury to the renal microvascular endothelium of rats with ROSC. 


\section{Materials and methods}

Animals and reagents. A total of 70 specific-pathogen free healthy male Sprague-Dawley rats (age, 12 to 14 weeks; weight, $370 \pm 20 \mathrm{~g}$ ) were purchased from Chengdu Dashuo Biological Technology Co., Ltd. (Chengdu, China). The rats were housed under a $12 / 12 \mathrm{~h} \mathrm{light/dark}$ cycle in a temperature- $\left(22 \pm 2^{\circ} \mathrm{C}\right)$ and humidity-(40 to $60 \%)$ controlled room with free access to fresh water and standard laboratory food. The procedures for animal care were approved by the Committee on the Ethics of Animal Experiments at Sichuan University (Sichuan, China).

The bipolar pacing electrode used for inducing ventricular fibrillation was the SelectSecure Model 3830 (Medtronic, Inc., Minneapolis, MN, USA). The PGE1 solution $(5 \mu \mathrm{g} / \mathrm{ml})$ was purchased from Beijing Tide Pharmaceutical Co., Ltd. (Beijing, China). The vascular endothelial (VE)-cadherin antibody (cat. no. \#2500) was purchased from Cell Signaling Technology, Inc. (Danvers, MA, USA). Vascular endothelial growth factor receptor (VEGFR) antibody (cat. no. \#BS4205) was purchased from Bioworld Technology, Inc. (St. Louis Park, MN, USA). The RNA prep pure, Quant cDNA and SuperReal PreMix Plus (SYBR-Green) kits were purchased from Tiangen Biotech Co., Ltd. (Beijing, China). The rat TM ELISA kit (cat. no. \#CSB-E07939r) was purchased from Cusabio Biotech Co., Ltd. (Wuhan, Hubei, China). The rat IL-6 (cat. no. \#ab00772) and TNF- $\alpha$ ELISA kit (cat. no. \#ab100785) were purchased from Abcam (Shanghai, China).

Animal model and experimental protocol. A total of 14 rats were included in each group. Rats without VF were considered to be the Sham group (S). VF was induced in the experimental rats by transesophageal cardiac pacing with an alternating current $(50 \mathrm{~Hz}, 6 \mathrm{~mA}, 4 \mathrm{msec})$ for $150 \mathrm{~s}(13,14)$. After $4 \mathrm{~min}$, the rats with cardiac arrest were resuscitated with 200 times/min chest compression using a home-made animal cardiopulmonary resuscitator. ROSC for the rats was defined as the return of supraventricular rhythm with a mean aortic pressure of $\geq 60 \mathrm{mmHg}$ for a minimum of $10 \mathrm{~min}$ (15). A total of 56 rats with successful cardiopulmonary resuscitation (CPR) were established as the ROSC model and then randomly divided into 4 groups: ROSC control group (R), PGE1 group [P; $1 \mathrm{ml}$ PGE1 solution was administered intravenously with a syringe pump in $1 \mathrm{~min}$ (high injection speed would decrease the success rate of resuscitation) ], TTM group ( $\mathrm{T}$; body temperature was decreased to $33 \pm 1^{\circ} \mathrm{C}$ within $30 \mathrm{~min}$ by placing ice around the rats) and PGE1/TTM group (PT; combined treatment with PGE1 and mild hypothermia). Blood samples were drawn from the left femoral vein of 5 rats from each group at $0.5,4$ and $8 \mathrm{~h}$ to evaluate the concentration of TM, TNF- $\alpha$ and IL-6. In every group, 3 rats were sacrificed at each time point $(0.5,4$ and $8 \mathrm{~h}$ ) for VE-cadherin and VCAM- 1 mRNA expression analysis with the renal tissue homogenate. At $8 \mathrm{~h}$ post treatment, hematoxylin and eosin (H\&E) and VE-cadherin/VEGFR double fluorescent immunohistochemistry (IHC) staining was performed (Fig. 1).

$H \& E$ staining. $\mathrm{H} \& \mathrm{E}$ staining was performed on tissue slides as described previously (16). Cell edema, inflammatory cell infiltration and micro-thrombus formation were examined by microscopy using the blind method. Tubular injury was scored

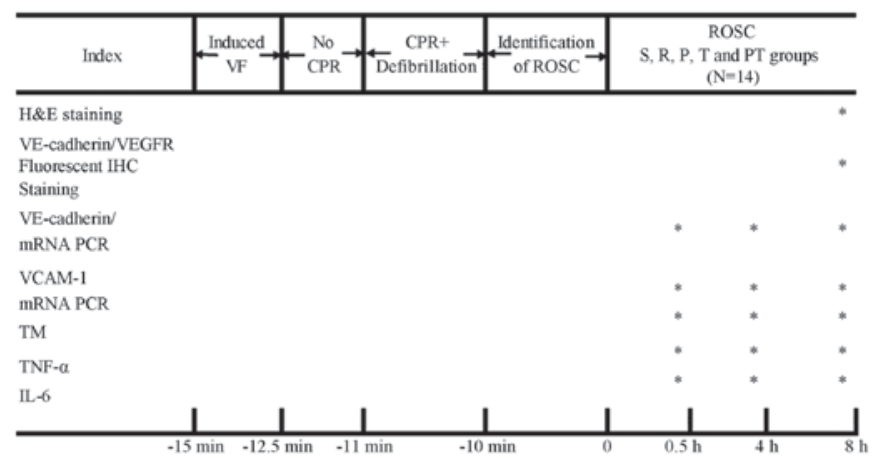

Figure 1. Schematic of the protocol and evaluation indexes. The asterisks (*) indicate which experimental analyses were performed at different time point. H\&E staining and VE-cadherin/VEGFR fluorescent IHC staining were evaluated at $8 \mathrm{~h}$ following ROSC. The expression of VE-cadherin and VCAM-1 mRNA, and the levels of TM, IL- 6 and TNF- $\alpha$ in the plasma were analyzed at $0.5,4$ and $8 \mathrm{~h}$ following the induction of ROSC. ROSC, return of spontaneous circulation; R, ROSC control group; P, prostaglandin E1 group; T, target temperature management group; PT, prostaglandin E1/target temperature management group; $\mathrm{S}$, sham group; VF, ventricular fibrillation; CPR, cardiopulmonary resuscitation; H\&E, hematoxylin and eosin staining; VE, vascular endothelial; VEGFR, vascular endothelial growth factor receptor; IHC, immunohistochemistry; PCR, polymerase chain reaction; TM, thrombomodulin; TNF- $\alpha$, tumor necrosis factor- $\alpha$; IL- 6 , interleukin- 6 .

according to Pallor's method (17): In total, 100 tubules from 10 different high power fields were scored. Higher scores represented more severe damage (maximum score/tubule was 10), with points given for the presence and extent of tubular epithelial cell flattening (1 point), brush border loss (1 point), cell membrane bleb formation ( 1 or 2 points), interstitial edema (1 point), cytoplasmic vacuolization (1 point), cell necrosis (1 or 2 points) and tubular lumen obstruction (1 or 2 points). Two technicians calculated these scores and the average values of the two sets of scores were recorded.

Fluorescent IHC staining. VE-cadherin/VEGFR double fluorescent IHC staining was performed according to the manufacturer's instructions (18). I/R injury to the RMEC was assessed by the extent of the damage exhibited by VE-cadherin immunostaining in the majority of the renal microvascular endothelium.

Reverse transcription-quantitative polymerase chain reaction $(R T-q P C R)$. Renal total RNA was extracted using the RNA prep pure kit according to manufacturer's instructions. The quantity of RNA product was determined using a UV-Visible spectrophotometer (Thermo Scientific Evolution 201; Thermo Fisher Scientific, Inc., Waltham, MA, USA) and the ratio of $28 \mathrm{~S}, 18 \mathrm{~S}$ and $5 \mathrm{~S}$ bands on a $2 \%$ agarose gel. Then, total RNA was reverse transcribed to cDNA using the Quant cDNA kit (Tiangen Biotech Co., Ltd.), according to the manufacturer's instructions. cDNA was used as a template for quantification of the genes of interest by their primers on the CFX-96 Touch Real-time System (Bio-Rad Laboratories, Inc., Hercules, CA, USA). The Super Real PreMix Plus (SYBR-Green) kit (Tiangen Biotech Co., Ltd.) was used for qPCR, according to the manufacturer's instructions. The thermocycling conditions for qPCR were as follows: $95^{\circ} \mathrm{C}$ for $15 \mathrm{~min}$, then 40 cycles of 


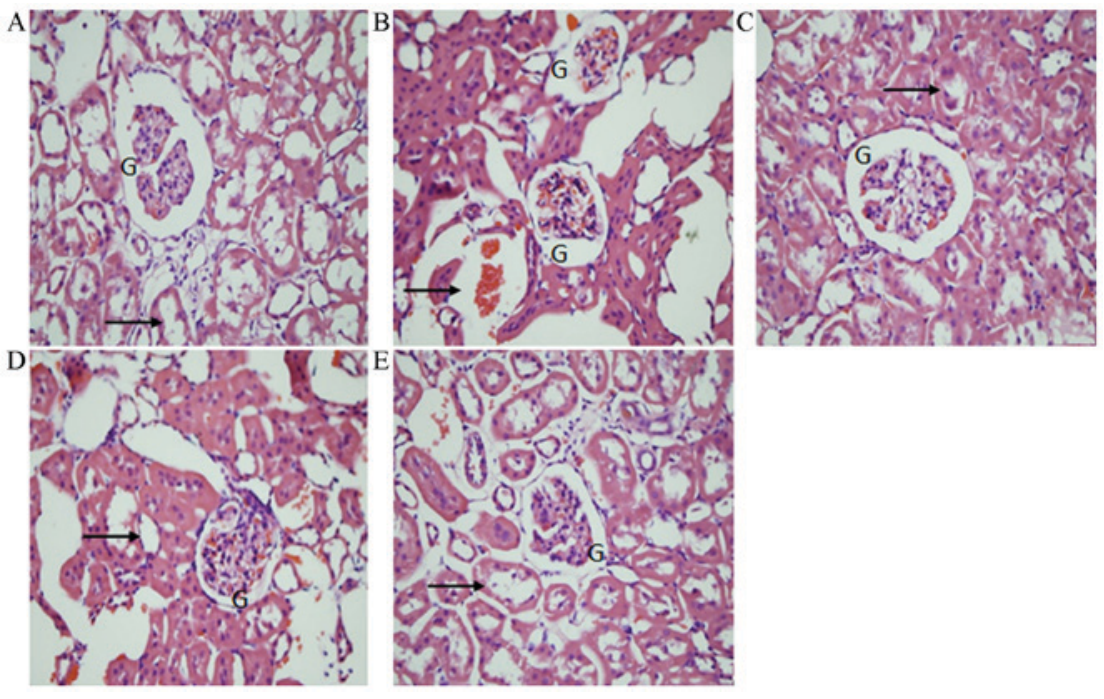

Figure 2. Hematoxylin and eosin staining of renal homogenate tissues (magnification, $x 400$ ). The black arrows indicate kidney tubules and 'G' represents renal glomeruli. (A) The sham group exhibited normal renal morphology. (B) The ROSC control group exhibited a distinctive pattern of ischemic injury (dilation of renal tubules, cast formation and tubular epithelial cell necrosis). (C) The renal architecture of the PGE1 group was relatively preserved, however, tubular cell swelling and inflammatory cell infiltration were also apparent. (D) The damage to the renal tubular structure exhibited by the TTM group was markedly alleviated when compared with the ROSC control group and the degree of cell swelling appeared to be marginally improved when compared with the PGE1 group. (E) The PGE1/TTM group had the lowest degree of vacuolization and cast formation, with almost no differences to the normal tissue. ROSC, return of spontaneous circulation; PGE1, prostaglandin E1; TTM, target temperature management.

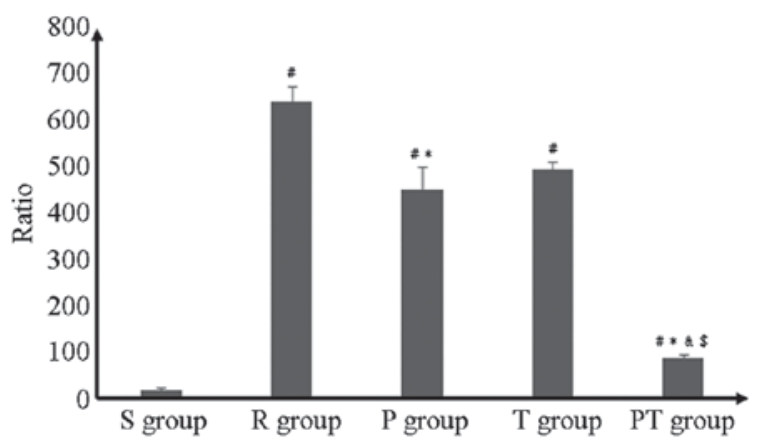

Figure 3. Tubular injury score for the R group differed from $\mathrm{S}$ group due to the extent of cellular necrosis, brush border loss, cell swelling and cast formation. P, T and PT groups also exhibited higher scores than the S group. Only the P and PT groups were significantly different when compared to the $R$ group $(P<0.05)$. There was no difference between the $\mathrm{P}$ and $\mathrm{T}$ groups. Data are presented as the mean \pm standard deviation. ${ }^{\prime \prime} \mathrm{P}<0.05$ vs. $\mathrm{S}$ group; ${ }^{*} \mathrm{P}<0.05$ vs. R group; ${ }^{\circledR} \mathrm{P}<0.05$ vs. $\mathrm{P}$ group; ${ }^{\$} \mathrm{P}<0.05$ vs. T group. $\mathrm{S}$, sham group; $\mathrm{R}$, return of spontaneous circulation control group; P, prostaglandin E1 group; $\mathrm{T}$, target temperature management group; PT, prostaglandin E1/target temperature management group.

$95^{\circ} \mathrm{C}$ for $10 \mathrm{sec}, 55^{\circ} \mathrm{C}$ for $20 \mathrm{sec}$ and $72^{\circ} \mathrm{C}$ for $30 \mathrm{sec}$. Actin served as the internal control. The primers used were as follows: VE-cadherin, forward, 5'-CATCCGCAAGACCAG TGAC-3' and reverse, 5'-ACCACGTCCTTGTCTGTTGC-3'; VACM-1, forward, 5'-TACATTGGCACCATCTCA-3' and reverse, 5'-GTTCAGCATCAGGGAGTT-3'; $\beta$-actin, forward, 5'-CCCATCTATGAGGGTTACGC-3' and reverse, 5'-TTT AATGTCACGCACGATTTC-3'.

ELISA. ELISA kits were used for the evaluation of TM, TNF- $\alpha$ and IL- 6 concentrations in the blood collected at 0.5 , 4 and $8 \mathrm{~h}$ for each group and were performed according to manufacturer's instructions.
Statistical analysis. Data are presented as mean \pm standard deviation. Comparisons between groups were analyzed by one-way analysis of variance. Comparisons between 2 groups with $\mathrm{P}<0.05$ were further analyzed by Student-Newman-Keuls analysis. Using Pearson's correlation analysis, the correlation between TM peak concentration at $8 \mathrm{~h}$ and the concentration of TNFa, and between TM peak concentration and the concentration of IL- 6 in each group was analyzed. P $<0.05$ was considered to indicate a statistically significant difference. All statistical analyses were performed using SPSS 19.0 software (IBM SPSS, Armonk, NY, USA).

\section{Results}

PGE1, TTM and PGE1/TTM combined interventions demonstrate different levels of protective effects on the renal tissue of rats with ROSC. Rat kidney sections were stained using $\mathrm{H} \& \mathrm{E}$ and examined using a light microscope (magnification, x400; Fig. 1). The morphology of the glomeruli and tubules was assessed. The normal structure was preserved in the S group, while a decrease in glomerulus size and widespread degeneration of the tubular architecture, intratubular cast formation and luminal congestion with extensive loss of the brush border were observed in the $\mathrm{R}$ group. The $\mathrm{P}$ and $\mathrm{T}$ groups exhibited marked improvements in histological features associated with renal injury. In the PT group, there was no significant difference when compared with the sham group (Fig. 2).

Blind review of the specimens from different groups revealed greater tubular injury in the $\mathrm{R}$ group. The $\mathrm{P}$ and $\mathrm{PT}$ groups exhibited a significant protective effect; the cytoplasmic vacuolization, cellular necrosis, tubular luminal debris and obstruction were much more marked in the $\mathrm{R}$ group $(\mathrm{P}<0.05)$. Statistical analysis revealed that the PT group experienced greater benefits with respect to reductions in injury than the $\mathrm{P}$ and $\mathrm{T}$ groups ( $\mathrm{P}<0.05$; Fig. 3$)$. 


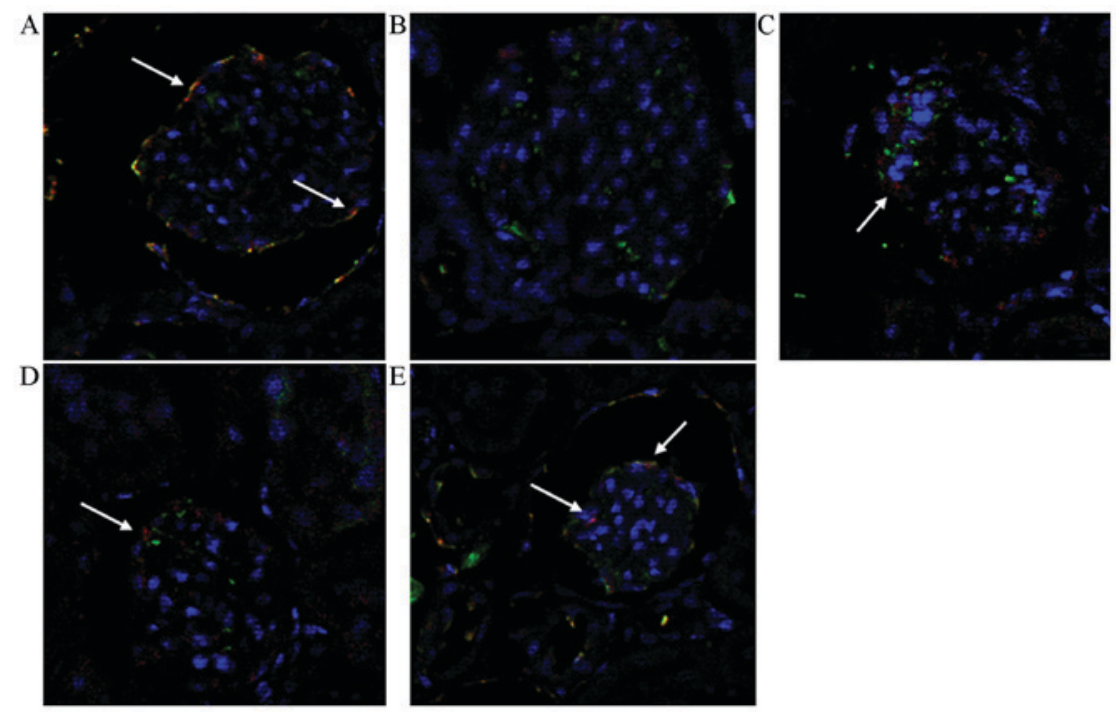

Figure 4. Double fluorescent immunohistochemistry staining in VEGFR/VE-cadherin (magnification, x400); VE-cadherin protein is shown in red and VEGFR (VEGFR positive, green) was used to label the RMEC. The white arrows indicate areas where the VE-cadherin protein is expressed normally at RMEC junctions (VEGFR/VE-cadherin positive, pink). (A) In the sham group, VE-cadherin protein was expressed normally in RMEC junctions. (B) In the ROSC control group, the expression significantly decreased. (C) Though the damage to VE-cadherin protein expression in the PGE1 group was still apparent, it was relatively lessened when compared with the ROSC group. (D) The TTM group exhibited similar results to the PGE1 group. (E) The PGE1/TTM group exhibited the highest expression among interventions. ROSC, return of spontaneous circulation; PGE1, prostaglandin E1; TTM, target temperature management; PT, PGE1/TTM group; VEGFR, vascular endothelial growth factor receptor; VE, vascular endothelial; RMEC, renal microvascular endothelium cells.

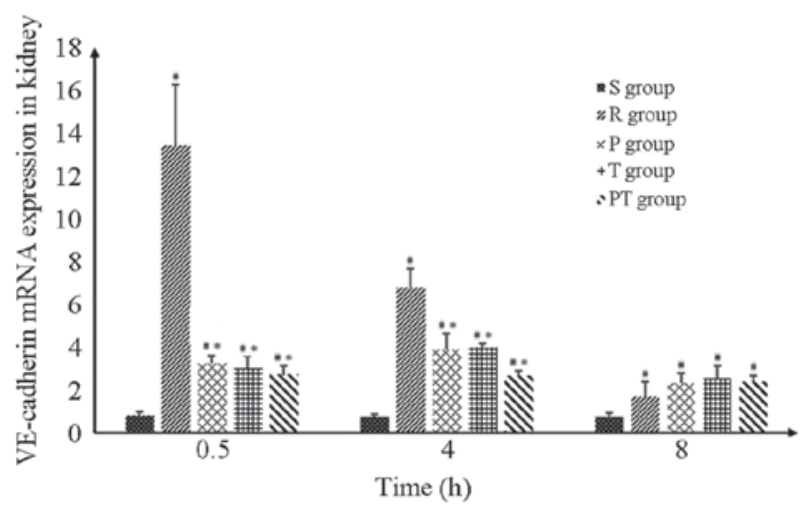

Figure 5. VE-cadherin mRNA expression. VE-cadherin mRNA levels markedly increased following return of spontaneous circulation induction and gradually declined within $8 \mathrm{~h}(\mathrm{P}<0.05)$. At 0.5 and $4 \mathrm{~h}$, there was a significant difference between the $\mathrm{R}$ group and the $\mathrm{P}$, $\mathrm{T}$ and $\mathrm{PT}$ groups $(\mathrm{P}>0.05)$. However, there was no statistical difference between the $\mathrm{R}$ group and the 3 intervention groups at $8 \mathrm{~h}$, although the VE-cadherin mRNA expression levels were still higher when compared to the $\mathrm{S}$ group. ${ }^{*} \mathrm{P}<0.05$, vs. $\mathrm{S}$ group; ${ }^{*} \mathrm{P}<0.05$ vs. $\mathrm{R}$ group. Data are presented as the mean \pm standard deviation. S, sham group; R, return of spontaneous circulation control group; P, prostaglandin E1 group; $\mathrm{T}$, target temperature management group; $\mathrm{PT}$, prostaglandin E1/target temperature management group; VE, vascular endothelial.

Benefits of PGE1, TTM and PGE1/TTM combined interventions for I/R injury to the renal microvascular endothelium in rats with ROSC. The kidney is a blood vessel-rich organ. The microvascular endothelial cells are primarily present in the glomerular and peritubular capillary bed. To determine the extent of the damage caused by I/R injury to the RMEC, the pattern of VE-cadherin immunostaining was examined. Under physiological conditions, VE-cadherin immunostaining was noted along the renal microvascular endothelium. Following $\mathrm{I} / \mathrm{R}$ injury, the loss of VE-cadherin immunostaining in the

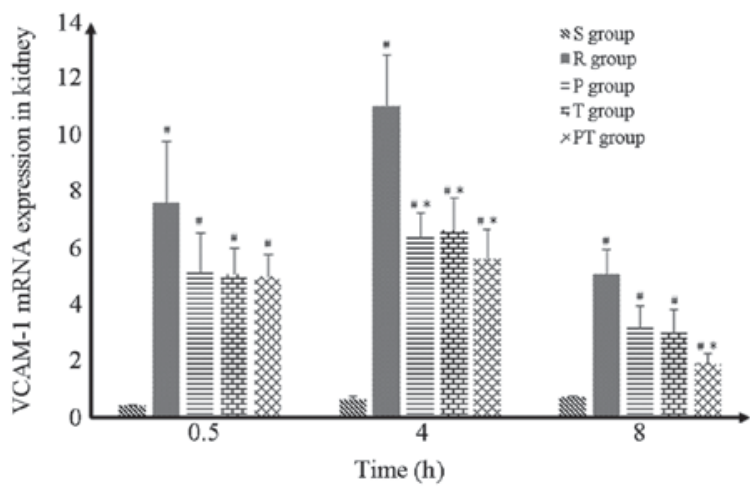

Figure 6. VCAM-1 mRNA expression. VCAM-1 mRNA expression increased at each time period. Following $4 \mathrm{~h}$ post return of spontaneous circulation induction, it peaked and, at the same time point, 3 interventions resulted in a significantly smaller increase in VCAM-1 mRNA expression. However, only the PT group exhibited a marked effect at $8 \mathrm{~h}$. ${ }^{\#} \mathrm{P}<0.05$ vs. $\mathrm{S}$ group; ${ }^{*} \mathrm{P}<0.05$ vs. $\mathrm{R}$ group. Data are presented as the mean \pm standard deviation. VCAM-1, vascular cell adhesion molecule-1; S, sham group; R, return of spontaneous circulation control group; P, prostaglandin E1 group; T, target temperature management group; PT, prostaglandin E1/target temperature management group.

majority of the renal microvascular endothelium suggested a disruption of the normal junctional complex. The destruction of VE-cadherin in the P, T and PT groups was markedly decreased compared with the R group, while the PT group was similar to the sham operation group under physiological conditions (Fig. 4).

Relative quantitative analysis of mRNA expression of VE-cadherin and VCAM-1 genes in renal tissue with different interventions was performed using RT-qPCR. In I/R injury, enhanced VE-cadherin mRNA expression was observed in the rat kidney within $8 \mathrm{~h}$ following ROSC. When compared to the ROSC control group, at the 0.5 and $4 \mathrm{~h}$ time points, the 3 intervention groups significantly slowed the rise of VE-cadherin 
Table I. Circulating thrombomodulin concentration at different time points following the return of spontaneous circulation.

TM concentration at indicated time point $(\mathrm{ng} / \mathrm{ml})$

\begin{tabular}{lrrr}
\cline { 2 - 4 } Group & $0.5 \mathrm{~h}$ & $4 \mathrm{~h}$ & $8 \mathrm{~h}$ \\
\hline S & $6.728 \pm 0.679$ & $7.218 \pm 0.832$ & $8.229 \pm 0.795$ \\
R & $15.753 \pm 0.509^{\mathrm{a}}$ & $17.877 \pm 0.432^{\mathrm{a}}$ & $20.364 \pm 0.504^{\mathrm{a}}$ \\
P & $11.653 \pm 1.153^{\mathrm{a}, \mathrm{b}}$ & $12.655 \pm 1.268^{\mathrm{a}, \mathrm{b}}$ & $13.503 \pm 0.357^{\mathrm{a}, \mathrm{b}}$ \\
T & $10.952 \pm 0.119^{\mathrm{a}, \mathrm{b}}$ & $11.866 \pm 0.722^{\mathrm{a}, \mathrm{b}}$ & $14.376 \pm 0.550^{\mathrm{a}-\mathrm{c}}$ \\
PT & $9.622 \pm 0.909^{\mathrm{a}-\mathrm{d}}$ & $9.298 \pm 0.314^{\mathrm{a}-\mathrm{d}}$ & $9.316 \pm 0.529^{\mathrm{b}-\mathrm{d}}$
\end{tabular}

${ }^{\mathrm{a}} \mathrm{P}<0.05$ vs. S group; ${ }^{\mathrm{b}} \mathrm{P}<0.05$ vs. $\mathrm{R}$ group; ${ }^{\mathrm{c}} \mathrm{P}<0.05$ vs. $\mathrm{P}$ group; ${ }^{\mathrm{d}} \mathrm{P}<0.05$ vs. $\mathrm{T}$ group. When compared to the $\mathrm{S}$ group, the concentrations of TM were markedly higher in the R, P, T and PT groups at almost every time point ( $\left.{ }^{\mathrm{a}} \mathrm{P}<0.05\right)$, except the PT group at $8 \mathrm{~h}(\mathrm{P}>0.05)$. Following ischemia/reperfusion injury, the $\mathrm{P}, \mathrm{T}$ and $\mathrm{PT}$ groups exhibited significant differences when compared to the $\mathrm{R}$ group at all time points ( ${ }^{\mathrm{b}} \mathrm{P}<0.05$ ). However, there was no significant difference between the $\mathrm{P}$ and $\mathrm{T}$ groups at 0.5 and $4 \mathrm{~h}(\mathrm{P}>0.05)$. By contrast, the PT group was significantly different to the $\mathrm{P}$ and $\mathrm{T}$ groups at all time points $\left({ }^{\mathrm{c}, \mathrm{d}} \mathrm{P}<0.05\right)$. Data are presented as the mean \pm standard deviation. TM, thrombomodulin; ROSC, return of spontaneous circulation; R, ROSC control group; P, prostaglandin E1 group; T, target temperature management group; PT, prostaglandin E1/target temperature management group; S, sham group.

Table II. Concentration of TNF- $\alpha$ and IL-6 at different time points following the return of spontaneous circulation.

Concentration at indicated time point $(\mathrm{ng} / \mathrm{ml})$

\begin{tabular}{lcccc} 
Parameters & Group & $0.5 \mathrm{~h}$ & $4 \mathrm{~h}$ & $8 \mathrm{~h}$ \\
\hline TNF- $\alpha$ & $\mathrm{S}$ & $222.198 \pm 6.903$ & $219.646 \pm 5.926$ & $219.506 \pm 3.552$ \\
& $\mathrm{R}$ & $219.986 \pm 5.679$ & $275.932 \pm 10.654^{\mathrm{a}}$ & $282.702 \pm 32.965^{\mathrm{a}}$ \\
& $\mathrm{P}$ & $216.464 \pm 9.078$ & $242.682 \pm 9.678^{\mathrm{a}, \mathrm{b}}$ & $232.376 \pm 11.187^{\mathrm{b}}$ \\
& $\mathrm{T}$ & $220.503 \pm 6.154$ & $257.123 \pm 10.215^{\mathrm{a}, \mathrm{b}}$ & $251.128 \pm 6.215^{\mathrm{a}}$ \\
& PT & $219.622 \pm 15.341$ & $233.776 \pm 6.094^{\mathrm{a}-\mathrm{c}}$ & $214.594 \pm 12.955^{\mathrm{b}, \mathrm{c}}$ \\
IL-6 & S & $78.968 \pm 8.840$ & $80.842 \pm 7.140$ & $83.852 \pm 4.217$ \\
& R & $85.094 \pm 7.719$ & $100.892 \pm 3.838^{\mathrm{a}}$ & $116.112 \pm 8.102^{\mathrm{a}}$ \\
& P & $84.868 \pm 4.713$ & $95.736 \pm 7.831$ & $97.686 \pm 10.606^{\mathrm{a}}$ \\
& T & $85.820 \pm 6.217$ & $93.432 \pm 5.429$ & $103.770 \pm 8.632^{\mathrm{a}}$ \\
& PT & $86.244 \pm 11.589$ & $89.946 \pm 5.476$ & $90.404 \pm 6.455^{\mathrm{b}}$ \\
\hline
\end{tabular}

${ }^{\mathrm{a}} \mathrm{P}<0.05$ vs. S group; ${ }^{\mathrm{b}} \mathrm{P}<0.05$ vs. $\mathrm{R}$ group; ${ }^{\mathrm{C}} \mathrm{P}<0.05$ vs. T group. At $0.5 \mathrm{~h}$ following ROSC induction, no significant differences were identified in TNF- $\alpha$ or IL- 6 concentrations. At 4 h, the TNF- $\alpha$ concentration in the R, P, T and PT groups, and the IL- 6 concentration in the R group significantly increased when compared to the $\mathrm{S}$ group $\left({ }^{\mathrm{a}} \mathrm{P}<0.05\right)$. However, the $\mathrm{P}, \mathrm{T}$ and $\mathrm{PT}$ groups exhibited significantly lower concentrations of TNF- $\alpha$ at $4 \mathrm{~h}$ when compared to the R group ( $\mathrm{P} P<0.05$ ). At $8 \mathrm{~h}$, neither the concentration of TNF- $\alpha$ or IL-6 in the PT group was significantly different to that of the $\mathrm{S}$ group $(\mathrm{P}>0.05)$. Data are presented as the mean \pm standard deviation. TNF- $\alpha$, tumor necrosis factor- $\alpha$; IL-6, interleukin-6; ROSC, return of spontaneous circulation; R, ROSC control group; P, prostaglandin E1 group; T, target temperature management group; PT, prostaglandin E1/target temperature management group; S, sham group.

mRNA expression $(\mathrm{P}<0.05)$. However, no statistical differences were identified between these 3 intervention groups (Fig. 5).

VCAM-1 mRNA expression was also examined to assess I/R injury to the RMEC following ROSC. When compared to the $\mathrm{S}$ group, all of the other groups exhibited significantly upregulated expression of VCAM-1 mRNA within $8 \mathrm{~h}(\mathrm{P}<0.05)$. When VCAM-1 mRNA expression rose to the highest level at $4 \mathrm{~h}$, the 3 different interventions demonstrated a marked ability to decrease the expression $(\mathrm{P}<0.05)$. There was no marked difference between the $\mathrm{R}$ group and $\mathrm{P}$ or $\mathrm{T}$ group at $8 \mathrm{~h}$; however, the $\mathrm{PT}$ treatment did significantly reduce the mRNA expression of VCAM 1 ( $\mathrm{P}<0.05$; Fig. 6$)$.
Circulating TM, a common early indicator of endothelial injury, was used as a cell marker in the present study. I/R injury induced a rapid increase in circulating TM concentration. All 3 of the interventions provided a protective effect, however, the PT group appeared to have a much greater advantage (Table I).

Possible association between the benefits from 3 interventions and anti-inflammatory mechanism. TNF- $\alpha$ and IL-6 concentrations were chosen as the parameters for the study of the anti-inflammatory mechanism. The concentrations did not alter significantly immediately following the I/R injury and ROSC. However, they gradually increased over time and 
peaked at $8 \mathrm{~h}$. The 3 interventions provided beneficial effects by preventing the elevation of TNF- $\alpha$ concentration following 4 h. However, only the PT group demonstrated a much greater protective effect in TNF- $\alpha$ and IL- 6 when compared with the other groups at $8 \mathrm{~h}$ (Table II).

Statistical analysis demonstrated that, at $8 \mathrm{~h}, \mathrm{I} / \mathrm{R}$ injury generated peak levels of circulating TM, TNF- $\alpha$ and IL-6 concentrations, and the interventions simultaneously induced the most significant effects. Pearson's correlation analysis demonstrated that peak TM levels were significantly associated with peak levels of TNF- $\alpha$ and IL-6 concentrations (Fig. 7).

\section{Discussion}

The majority of studies on organ dysfunction following resuscitation from CA have focused on the brain and heart. The prevalence of extra-cerebral or cardiac organ injury and its impact on outcomes has been less well-characterized. A previous study highlighted acute kidney injury (AKI) in patients with CA; the frequency of AKI development following CA ranged from 12 to $43 \%$ and $>75 \%$ of these episodes occurred within 3 days following CA (19). Following more in-depth research, a number of controversies arose regarding some aspects, including whether AKI has an impact on hospital survival rates $(20,21)$ and whether AKI is associated with a lower probability of favorable neurological outcomes $(19,22)$. In the present study, RMEC was chosen as the target as the kidney is rich in micro-vessels and endothelial involvement in CPR as a primary or secondary target has been described previously in animal models and human patients (2).

Following ROSC, the systemic I/R response may lead to PCAS, which is characterized by the activation of immunological and coagulation pathways, and the release of inflammatory mediators, all of which lead to tissue hypoperfusion and multiple organ dysfunction (3). Specifically, systemic inflammation promotes disorders in the microcirculation due to metabolic imbalance, leukocyte activation, endothelial toxicity, and impairment of mitochondrial respiratory chain activity (23). Increases in pro-inflammatory cytokines and soluble receptors have been reported in patients $\mathrm{h}$ following resuscitation from CA (24). TNF- $\alpha$ and IL-6 are involved in systemic inflammation and stimulate the acute phase reaction. They are primarily involved in the regulation of immune cells and are also implicated in the induction of fever, apoptosis and the inhibition of cellular reproduction (25). Consistent with the present study, the immediate reperfusion period after ROSC following CA has previously been revealed to be characterized by an abrupt increase in plasma TNF- $\alpha$ and IL-6 (26). A previous study reported that early IL-6 levels are strongly associated with mortality and appear to be excellent predictors of the outcome of CA (12). However, the use of inflammation biomarkers as an indicator following $\mathrm{CA}$ is limited due to their poor specificity for $\mathrm{I} / \mathrm{R}$ injury and their exact mechanism and association with extensive endothelial damage is still unclear. The present study used TM to associate the inflammation markers with endothelial injury. TM is a transmembrane glycoprotein expressed on the surface of all vascular endothelial cells. Expression of TM is tightly regulated to maintain homeostasis and to ensure a
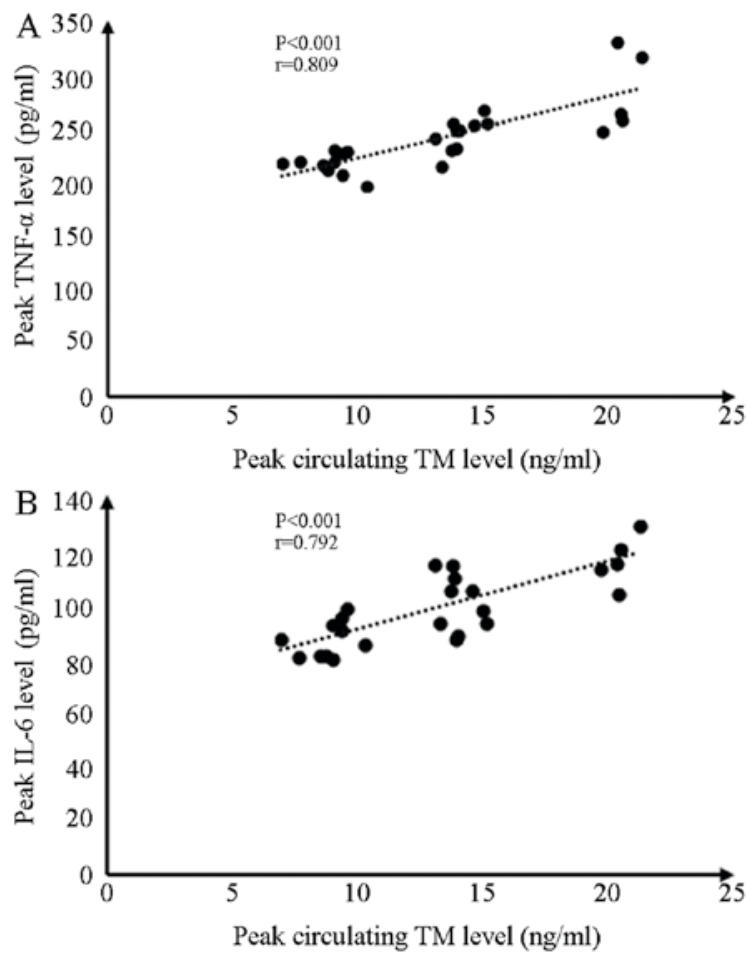

Figure 7. Correlation of peak TM levels with peak TNF- $\alpha$ and IL- 6 concentrations. The Pearson's correlation coefficients for (A) TNF- $\alpha$ and (B) IL-6 were 0.809 and 0.792 , respectively, which demonstrated strong linear relationships. TM, thrombomodulin; TNF- $\alpha$, tumor necrosis factor- $\alpha$; IL-6, interleukin-6.

rapid and localized hemostatic and inflammatory response to injury (27). The results of the correlation analysis revealed that the peak TM levels and the peak levels of the TNF- $\alpha$ and IL-6 concentrations are positively associated. This may indicate that the inflammatory response is the pathway for endothelial $\mathrm{I} / \mathrm{R}$ injury in the early stages following ROSC. Therefore, anti-inflammatory interventions may be effective therapeutic strategies.

PGE1 is rarely used in PCAS patients, however, its anti-inflammatory effect has been confirmed in other I/R injury models, particularly in the kidney (28). Recent studies have indicated that PGE1 may have a protective role as it blocks chemoattractant factors and weakens leukocyte adhesion to the microvascular endothelium, with lower tissue expression of some inflammatory markers involved in this process, potentially leading to cytoprotective activity $(29,30)$. The pathomorphological and ELISA results demonstrated that PGE1 reduced I/R injury to the RMEC following ROSC and markedly inhibited the release of TM (at 3 time points) and TNF- $\alpha$ (at 4 and $8 \mathrm{~h}$ ). In addition, the results from the VE-cadherin and VCAM-1 mRNA expression analysis indicated that PGE1 was able to effectively inhibit the rapid elevation of this mRNA expression, which may imply the endogenous compensatory requirements for rapid and severe $\mathrm{I} / \mathrm{R}$ damage to the endothelial cell junction (31).

TTM is already a fundamental part of the treatment for unconscious survivors of OHCA due to landmark studies that concluded that mild hypothermia $\left(32-34^{\circ} \mathrm{C}\right)$ improved survival and neurological outcome $(9,32,33)$. As survivors of OHCA exhibit a 'sepsis like syndrome' (3), anti-inflammation 
effects are thought to be a potential mechanism of TTM (34). In the present study, TTM alleviated I/R injury to the RMEC, decreased VE-cadherin and VCAM-1 mRNA expression, and suppressed the circulating TM concentration, which is similar to PGE1. However, TTM did not exhibit an apparent influence on inflammation biomarkers as there was no significant difference between the ROSC group and TTM group during the majority of the present study, except for the altered TNF- $\alpha$ levels at $4 \mathrm{~h}$. This may support the idea that TTM has a protective effect with respect to I/R injury to the endothelium, however, further research is required to confirm whether anti-inflammatory effects are the exact mechanism by which TTM improves the outcome during the early stages of ROSC.

The present study focused on the combined intervention of PGE1 and TTM. To the best of our knowledge, this has not been studied previously. A number of medications are normally given to patients with PCAS, in the same way they are for any other critically ill patient. The influence of body temperature on physicochemical properties of drug disposition, potentially contributing to drug-therapy and drug-disease interaction, should be considered when TTM is used for PCAS (35). The results demonstrated that the PGE1/TTM combined intervention had more beneficial effects compared with the single interventions in protecting the RMEC from I/R injury in CA, with significant inhibition of VE-cadherin protein loss, rapid promotion of VCAM-1 mRNA expression and TM release. Only TTM/PGE1 combined interventions effectively reduced the concentration of TNF- $\alpha$ and IL- 6 at $8 \mathrm{~h}$, producing similar results to the sham operation group. TTM and PGE1 interventions alone did not achieve this effect.

Due to limitations in experimental facilities, the present study used an alternating current delivered through transesophageal cardiac pacing instead of delivery to the classic right ventricular endocardium to induce the VF. The present study therefore differs from others to some extent with respect to current stimulation parameters and VF duration time. Further research is required to determine the response of proteins by using quantitative analysis.

In conclusion, the present study considered RMEC to be a useful indicator of general I/R injury following ROSC. Anti-inflammatory interventions may be one of the most promising options for future endothelial I/R injury treatment in the early phase of PCAS. The PGE1 and TTM interventions had protective effects with respect to I/R injury to the RMEC, while PGE1 exhibited a greater impact on inflammation markers than TTM. The PGE1/TTM combined intervention may exhibit an improved synergistic effect as an anti-inflammatory treatment compared with the single interventions.

\section{Acknowledgements}

The present study was supported by funding from the Transformation of Scientific and Technological Achievements of Chengdu Grant (grant no. 11DXYB294SF-027). The authors of the present study would like to thank the staff of the Transplantation Engineering and Immunology Laboratory of the West China Hospital of Sichuan University(Chengdu, Sichuan) for their technical help and constructive criticism.

\section{References}

1. Roberts BW and Trzeciak S: Systemic inflammatory response after cardiac arrest: Potential target for therapy? Crit Care Med 43: 1336-1337, 2015.

2. Adams JA: Endothelium and cardiopulmonary resuscitation. Crit Care Med 34 (12 Suppl): S458-S465, 2006.

3. Adrie C, Laurent I, Monchi M, Cariou A, Dhainaou JF and Spaulding C: Post resuscitation disease after cardiac arrest: A sepsis-like syndrome? Curr Opin Crit Care 10: 208-212, 2004.

4. Soares BL, Freitas MA, Montero EF, Pitta GB and Miranda F Jr: Alprostadil attenuates inflammatory aspects and leucocytes adhesion on renal ischemia and reperfusion injury in rats. Acta Cir Bras 29 (Suppl 2): S55-S60, 2014.

5. Vargas AV, Krishnamurthi V, Masih R, Robinson AV and Schulak JA: Prostaglandin E1 attenuation of ischemic renal reperfusion in the rat. J Am Coll Surg 180: 713-717, 1995.

6. Gupta PC, Matsushita M, Oda K, Nishikimi N, Sakurai T and Nimura Y: Attenuation of renal ischemia-reperfusion injury in rats by allopurinol and prostaglandin E1. Eur Surg Res 30: 102-107, 1998.

7. Huk I, Brovkovych V, Nanobashvili J, Neumayer C, Polterauer P, Prager M, Patton S and Malinski T: Prostaglandin E1 reduces ischemia/reperfusion injury by normalizing nitric oxide and superoxide release. Shock 14: 234-242, 2000.

8. Hong JP, Chung YK and Chung SH: The effect of prostaglandin E1 versus ischemia-reperfusion injury of musculocutaneous flaps. Ann Plast Surg 47: 316-321, 2001.

9. Callaway CW, Donnino MW, Fink EL, Geocadin RG, Golan E, Kern KB, Leary M, Meurer WJ, Peberdy MA, Thompson TM and Zimmerman JL: Part 8: Post-cardiac arrest care: 2015 American Heart Association Guidelines Update for Cardiopulmonary Resuscitation and Emergency Cardiovascular Care. Circulation 132 (18 Suppl 2): S465-S482, 2015.

10. Fries M, Stoppe C, Brücken D, Rossaint R and Kuhlen R: Influence of mild therapeutic hypothermia on the inflammatory response after successful resuscitation from cardiac arrest. J Crit Care 24: 453-457, 2009.

11. Bisschops LL, van der Hoeven JG, Mollnes TE and Hoedemaekers CW: Seventy-two h of mild hypothermia after cardiac arrest is associated with a lowered inflammatory response during rewarming in a prospective observational study. Crit Care 18: 546, 2014.

12. Bro-Jeppesen J, Kjaergaard J, Wanscher M, Nielsen N, Friberg H, Bjerre M and Hassager C: Systemic inflammatory response and potential prognostic implications after out-of-hospital cardiac arrest: A sub-study of the target temperature management trial. Crit Care Med 43: 1223-1232, 2015.

13. Chen MH, Liu TW, Xie L, Song FQ, He T, Zeng ZY and Mo SR: Ventricular fibrillation induced by transoesophageal cardiac pacing: A new model of cardiac arrest in rats. Resuscitation 74: 546-551, 2007.

14. Zhou J, Huang GQ, Li XM and Li XG: Effects of different stimulating parameters and their various combinations on transoesophageal electrical stululation induced cardiac arrest in rats. China J Emergency Resuscitaion and Disaster Med 8: 596-598, 2013.

15. Idris AH, Becker LB, Ornato JP, Hedges JR, Bircher NG, Chandra NC, Cummins RO, Dick W, Ebmeyer U, Halperin HR, et al: Utstein-style guidelines for uniform reporting of laboratory CPR research. Resuscitation 33: 69-84, 1996.

16. Cardiff RD, Miller $\mathrm{CH}$ and Munn RJ: Manual hematoxylin and eosin staining of mouse tissue sections. Cold Spring Harb Protoc 2, 665-668, 2014.

17. Pallor MS, Hoidal JR and Ferris TF: Oxygen free radicals in ischemic acute renal failure in the rat. J Clin Inves 74: 1156-1164, 1984.

18. Garvard J and Gutkind JS: VEGF controls endothelial permeability by promoting the beta-arrestin-dependent endocytosis of VE-cadherin. Nat Cell Biol 8: 1223-1234, 2006.

19. Tujjar O, Mineo G, Dell'Anna A, Poyatos-Robles B, Donadello K, Scolletta S, Vincent JL and Taccone FS: Acute kidney injury after cardiac arrest. Critical Care 19: 169, 2015.

20. Yanta J, Guyette FX, Doshi AA, Callaway CW and Rittenberger JC; Post Cardiac Arrest Service: Renal dysfunction is common following resuscitation from out-of-hospital cardiac arrest. Resuscitation 84: 1371-1374, 2013. 
21. Geri G, Guillemet L, Dumas F, Charpentier J, Antona M, Lemiale V, Bougouin W, Lamhaut L, Mira JP, Vinsonneau C and Cariou A: Acute kidney injury after out-of-hospital cardiac arrest: Risk factors and prognosis in a large cohort. Intensive Care Med 41: 1273-1280, 2015.

22. Hasper D, von Haehling S, Storm C, Jörres A and Schefold JC: Changes in serum creatinine in the first $24 \mathrm{~h}$ after cardiac arrest indicate prognosis: An observational cohort study. Crit Care 13: R168, 2009.

23. Huet O, Dupic L, Batteux F, Matar C, Conti M, Chereau C, Lemiale V, Harrois A, Mira JP, Vicaut E, et al: Postresuscitation syndrome: Potential role of hydroxyl radical-induced endothelial cell damage. Crit Care Med 39: 1712-1720, 2011.

24. Adrie C, Adib-Conquy M, Laurent I, Monchi M, Vinsonneau C, Fitting C, Fraisse F, Dinh-Xuan AT, Carli P, Spaulding C, et al: Successful cardiopulmonary resuscitation after cardiac arrest as a 'sepsis-like' syndrome. Circulation 106: 562-568, 2002.

25. Scolletta S, Donadello K, Santonocito C, Franchi F and Taccone FS: Biomarkers as predictors of outcome after cardiac arrest. Expert Rev Clin Pharmacol 5: 687-699, 2012.

26. Samborska-Sablik A, Sablik Z and Gaszynski W: The role of the immuno-inflammatory response in patients after cardiac arrest. Arch Med Sci 7: 619-626, 2011.

27. Conway EM: Thrombomodulin and its role in inflammation. Semin Immunopathol 34: 107-125, 2012.

28. Vargas AV, Krishnamurthi V, Masih R, Robinson AV and Schulak JA: Prostaglandin E1 attenuation of ischemic renal reperfusion injury in the rat. J Am Coll Surg 180: 713-717, 1995.

29. Kawamura T, Nara N, Kadosaki M, Inada $K$ and Endo $S$ : Prostaglandin E1 reduces myocardial reperfusion injury by inhibiting proinflammatory cytokines production during cardiac surgery. Crit Care Med 28: 2201-2208, 2000
30. Soares BL, Freitas MA, Montero EF, Pitta GB and Miranda F Jr: Alprostadil attenuates inflammatory aspects and leucocytes adhesion on renal ischemia and reperfusion injury in rats. Acta Cir Bras 29 (Suppl 2): S55-S60, 2014.

31. Chen F, Kondo N, Sonobe M, Fujinaga T, Wada H and Bando T: Expression of endothelial cell-specific adhesion molecules in lungs after cardiac arrest. Interact Cardiovasc Thorac Surg 7: 437-440, 2008

32. Hypothermia after Cardiac Arrest Study Group. Mild therapeutic hypothermia to improve the neurologic outcome after cardiac arrest. N Engl J Med 346: 549-556, 2002.

33. Bernard SA, Gray TW, Buist MD, Jones BM, Silvester W, Gutteridge $\mathrm{G}$ and Smith K: Treatment of comatose survivors of out-of-hospital cardiac arrest with induced hypothermia. N Engl J Med 346: 557-563, 2002.

34. Bro-Jeppesen J, Kjaergaard J, Wanscher M, Nielsen N, Friberg H, Bjerre $M$ and Hassager $C$ : The inflammatory response after out-of-hospital cardiac arrest is not modified by targeted temperature management at $33^{\circ} \mathrm{C}$ or $36^{\circ} \mathrm{C}$. Resuscitation 85: 1480-1487, 2014.

35. Zhou J and Poloyac SM: The effect of therapeutic hypothermia on drug metabolism and drug response: Cellular mechanisms to organ function. Expert Opin Drug Metab Toxicol 7: 803-816, 2011. 